\title{
SIRT1 Inhibition Affects Angiogenic Properties of Human MSCs
}

\author{
Botti Chiara, ${ }^{1,2}$ Caiafa Ilaria, ${ }^{1}$ Coppola Antonietta, ${ }^{1}$ Cuomo Francesca, ${ }^{1}$ \\ Miceli Marco, ${ }^{3}$ Altucci Lucia, ${ }^{1,3}$ and Cobellis Gilda ${ }^{1,2}$ \\ ${ }^{1}$ Department of Biochemistry, Biophysics and General Pathology, Second University of Napoli, Via L. De Crecchio 7, \\ 80138 Napoli, Italy \\ ${ }^{2}$ Istituto Nazionale Tumori, Struttura Complessa Oncologia Medica Melanoma Immunoterapia Oncologica e Terapia Innovativa, \\ Via Mariano Semmola, 80131 Napoli, Italy \\ ${ }^{3}$ Institute of Genetics and Biophysics "A. Buzzati-Traverso", CNR, Via P. Castellino 111, 80131 Napoli, Italy
}

Correspondence should be addressed to Botti Chiara; chiara_botti@yahoo.it and Cobellis Gilda; g.cobellis@unina2.it

Received 18 July 2014; Revised 7 August 2014; Accepted 8 August 2014; Published 27 August 2014

Academic Editor: Zongjin Li

Copyright (C) 2014 Botti Chiara et al. This is an open access article distributed under the Creative Commons Attribution License, which permits unrestricted use, distribution, and reproduction in any medium, provided the original work is properly cited.

\begin{abstract}
Human mesenchymal stem cells (hMSCs) are attractive for clinical and experimental purposes due to their capability of self-renewal and of differentiating into several cell types. Autologous hMSCs transplantation has been proven to induce therapeutic angiogenesis in ischemic disorders. However, the molecular mechanisms underlying these effects remain unclear. A recent report has connected MSCs multipotency to sirtuin families, showing that SIRT1 can regulate MSCs function. Furthermore, SIRT1 is a critical modulator of endothelial angiogenic functions. Here, we described the generation of an immortalized human mesenchymal bone marrowderived cell line and we investigated the angiogenic phenotype of our cellular model by inhibiting SIRT1 by both the genetic and pharmacological level. We first assessed the expression of SIRT1 in hMSCs under basal and hypoxic conditions at both RNA and protein level. Inhibition of SIRT1 by sirtinol, a cell-permeable inhibitor, or by specific sh-RNA resulted in an increase of prematuresenescence phenotype, a reduction of proliferation rate with increased apoptosis. Furthermore, we observed a consistent reduction of tubule-like formation and migration and we found that SIRT1 inhibition reduced the hypoxia induced accumulation of HIF-1 $\alpha$ protein and its transcriptional activity in hMSCs. Our findings identify SIRT1 as regulator of hypoxia-induced response in hMSCs and may contribute to the development of new therapeutic strategies to improve regenerative properties of mesenchymal stem cells in ischemic disorders through SIRT1 modulation.
\end{abstract}

\section{Introduction}

Human mesenchymal stem cells (hMSCs) have become an important tool for cell-based strategies. They can differentiate into a variety of cell types such as muscle, neural precursors, cardiomyocytes, and perivascular cells and are currently being tested in several approved clinical trials [1]. hMSCs can improve myocardial remodeling in infarcted heart [2] or promote angiogenesis in critical limb ischemia [3], due to their capacity to stimulate endothelial progenitor cells. Furthermore, hMSCs support neoangiogenesis also by releasing soluble factors that stimulate angiogenesis [4-9]. However, the molecular mechanisms of beneficial effects from hMSCsbased therapy remain unclear.
A recent report showed that SIRT1 might regulate MSCs function, providing a connection between sirtuin families and MSCs multipotency [10].

Sirtuins are classified as class III histone deacetylases (HDACs) [11], originally identified in yeast. They modulate a wide range of biological processes, spanning from DNA repair and oxidative stress responses to energy metabolism. Sirtuins activity is controlled by the cellular $[\mathrm{NAD}+] /$ $[\mathrm{NADH}]$ ratio, where NAD+ works as an activator, whereas nicotinamide and NADH act as inhibitors.

In mammals, the sirtuin family comprises seven members (SIRT1-SIRT7) with different biological functions and subcellular localizations [12-14]. SIRT1, SIRT6, and SIRT7 are mainly nuclear, whereas SIRT2 is found primarily in the 
cytosol. SIRT3, SIRT4, and SIRT5 are mitochondrial proteins [12]. Sirtuins are generally known to regulate the acetylation levels and the activity of histone and nonhistone regulatory proteins.

To date, sirtuins have emerged as potential therapeutic targets for treatment of human pathologies such as cardiovascular disease, inflammation, and cancer [15]. SIRT1 is the most studied member of sirtuins. It acts in various cellular processes and exerts its action activating and deactivating factors such as NF- $\kappa \mathrm{B}, \mathrm{p} 53, \mathrm{p} 73, \mathrm{SOD}$, and hypoxia-inducible transcription factors (HIFs) [16-19].

Since SIRT1 targets several proteins in distinct signaling pathways, modulation of SIRT1 activity alters the biological activity of entire signaling networks modifying disease progression, such as pathological angiogenesis or atherosclerosis.

An important component of pathological angiogenesis is represented by hypoxia that alters the cellular redox state and activates SIRT1. In addition, hypoxia works through multiple pathways to regulate angiogenesis, for instance, through the modulation of secreted angiogenic proteins, such as vascular endothelial growth factor (VEGF), stimulated by increased expression of transcription factors such as HIF-1 $\alpha$ [20].

Furthermore, a "protective" role of SIRT1 in endothelial cells was described [21, 22]. A recent report has investigated the function of SIRT1 in regulating the differentiation of mesenchymal stem cells by deacetylating $\beta$-catenin in mice [10]. Several studies demonstrated that inhibition of SIRT1 impairs cell growth in cancer cells $[23,24]$. Gorenne et al. [25] reported that SIRT1 expression was reduced in human atherosclerotic plaques and in vascular smooth muscle cells. However, to the best of our knowledge, the effects of SIRT1 modulation on angiogenesis in hMSCs have not been studied yet.

In this study, we described the generation of an immortalized human mesenchymal bone marrow-derived cell line $(\mathrm{MeBM})$ and we investigated whether SIRT1 has an effect on angiogenic capability of hMSCs by inhibiting SIRT1 through pharmacological and genetic approaches.

Since recent studies have identified SIRT1 as a critical modulator of angiogenesis $[18,26]$, we tested whether the inhibition of SIRT1 activity is associated with the reduction of angiogenic ability of hMSCs and impaired hypoxic response in these settings. We found that the inhibition of SIRT1 activity resulted in reduced capacity to proliferate, to migrate, and to form three-dimensional networks of vessel-like structures. In addition, SIRT1 inhibition reduced the hypoxia-induced accumulation of HIF- $1 \alpha$ and its transcriptional activity in hMSCs.

Our results suggested that SIRT1 is involved in angiogenic response of hMSCs in vitro and modulates the hypoxic response through inhibiting HIF- $1 \alpha$ activity.

Our findings may help to understand the role of SIRT1 in hMSCs in promoting angiogenesis and may contribute to the development of new strategies to improve the hMSCs-based regenerative effects by modulating SIRT1 activity.

\section{Methods}

2.1. Reagents. Sirtinol was purchased from Selleck Chemicals LLC (Houston, TX, USA). Culture medium and its supplements including antibiotics and fetal bovine serum (FBS) were purchased from Euroclone (Italy). Primary antibodies against SIRT1 (Abcam, Cambridge, UK), HIF- $1 \alpha$ (Santa Cruz Biotechnology, Santa Cruz, CA, USA), and tubulin (SigmaAldrich, Milan, Italy) were used. Sirtinol was dissolved in dimethyl sulfoxide (DMSO, Sigma-Aldrich) to the appropriate concentrations according to reported procedures. DMSO was also present in the corresponding control.

2.2. Cell Lines and Culture Medium. Human mesenchymal stem cells (hMSCs) were obtained from bone marrow as described by Cobellis et al. [8]. Cells were plated in RPMI 1640 growth medium (Euroclone SPA, Italy), containing 10\% heat-inactivated FBS, 1\% Pen-strep, and 1\% L-Glutamine.

Cells were maintained as monolayers in a humidified atmosphere containing $5 \% \mathrm{CO}_{2}$ at $37^{\circ} \mathrm{C}$ and the culture medium was replaced every two days.

Hypoxic culture conditions were achieved in a BD GasPak EZ Anaerobe Gas Generating Pouch System (BD Biosciences, San Diego). As certified by the manufacturer, the Anaerobe Gas Generating Pouch System produces an atmosphere containing $10 \%$ carbon dioxide and $1 \%$ oxygen.

Starvation conditions were obtained incubating cells in RPMI 1640 containing $0.2 \%$ FBS.

2.3. Infection. After plating, bone marrow cells were grown to confluence and coinfected with HPV16 E6/E7 and hTERT lentiviral vectors (infection number 1). After a week the cells were split and infected again only with hTERT (infection number 2) and cultured until stabilization. Samples were observed and photographed with DMI 6000 inverted microscope (Leica Microsystems) using Leica LAS Image Analysis software (Leica Microsystems). The protocol was also reported in Miceli et al. [27].

2.4. hPV16 E6/E7 and hTERT Lentiviral Production. HIVlbased SIN lentiviral vectors were derived from SINF-MU3W-S vector backbone. hPV16 E6/E7 was inserted upstream of an encephalomyocarditis virus internal ribosome entry site- (IRES-) yellow fluorescent protein (YFP) gene cassette into SINF-MU3-W-S to generate SINF-MU3-E6E7-IRESYFPW-S. SINF-MU3-hTERT-IRES-GFPW-S was generated by inserting hTERT cDNA upstream of an IRES-green fluorescent protein (GFP) gene cassette into SINF-MU3-W-S. VSVG-pseudotyped lentiviral vectors were generated in $150 \mathrm{~mm}$ tissue culture dishes by transient cotransfection with (1) VSVG-expressing construct pCMV-VSV-G (Invitrogen, USA) $(66 \mu \mathrm{g})$, (2) packaging construct pCMV $\Delta \mathrm{R} 8.2$ (addgene) $(48 \mu \mathrm{g})$, and (3) lentiviral vector plasmids (pSin hTERT or Psin E6-E7) $(66 \mu \mathrm{g})$ into subconfluent HEK 293FT cells (Invitrogen) by calcium phosphate precipitation (Clontech, Calphos Mammalian Transfection Kit). The supernatant containing the virus was produced in HEK-293FT, collected, filtered, and used to infect bone marrow cells. 
2.5. Gene Knockdown Using Lentiviral Vector. Cells $\left(10^{5}\right)$ were grown in RPMI 1640 medium $4.5 \mathrm{~g} / \mathrm{L}$ glucose (Euroclone SPA, Italy) supplemented with $20 \%$ FBS (Euroclone SPA, Italy), $100 \mathrm{U} / \mathrm{mL}$ Pen-strep (Lonza Group Ltd), and $2 \mathrm{mM}$ L-Glutamine (Lonza Group Ltd) at $37^{\circ} \mathrm{C}$, in $5 \% \mathrm{CO}_{2}$ fully humidified atmosphere. Cells were first grown for $24 \mathrm{~h}$ and then infected with the rLV.H1.sh2Sirt1.EF1.GFP Lentivirus, with a $2.5 \mathrm{MOI}$, overnight as described by Miceli et al. [27].

2.6. RNA Extraction and $q P C R$. Total RNA was isolated from hMSCs by miRNeasy Mini kit (QIAGEN GE). $500 \mathrm{ng}$ was converted to cDNA using the Quantitect Reverse Transcription kit (QIAGEN GE).

qPCR assays were performed using an iCycler (BioRad Laboratories, USA) and the Sybergreen Super mix (BioRad Laboratories, USA). The primer sequences and qPCR conditions are available on request.

2.7. Protein Extraction and Western Blot Analysis. hMSCs were incubated with $100 \mu \mathrm{M}$ sirtinol for $24 \mathrm{~h}$ and then we collected lysates from cells exposed to $1 \% \mathrm{O}_{2}$ for $6 \mathrm{~h}$.

Cells were lysed in buffer containing $20 \mathrm{mM}$ Tris $\mathrm{HCl}$, $100 \mathrm{mM} \mathrm{NaCl}, 10 \mathrm{mM} \mathrm{MgCl} 2,1 \% \mathrm{NP} 40,10 \%$ glycerol, $0.1 \mathrm{M}$ $\mathrm{NaF}, 100 \mu \mathrm{M}$ sodium vanadate, and protease inhibitors mixture (Roche LTD, GE). Equal amounts of supernatant were separated by SDS-polyacrylamide gels. Proteins were transferred to nitrocellulose membranes (WhatmanProtran, GE Healthcare) and membranes were blocked with blocking buffer (TBS-Tween buffer containing 5\% milk). Subsequently, the membranes were incubated with primary antibodies at $4^{\circ} \mathrm{C}$ overnight. After three washes for $10^{\prime}$ with TTBS buffer $(50 \mathrm{mM}$ Tris $\mathrm{HCl}, \mathrm{pH} 8,150 \mathrm{mM} \mathrm{NaCl}$, and $0.5 \%$ Tween-20), the membranes were incubated with horseradish peroxidase-conjugated anti-mouse or anti-rabbit antibody ( $1: 10.000$, Santa Cruz Biotechnology, Santa Cruz, CA, USA) for $1 \mathrm{~h}$ at room temperature and then washed for $30 \mathrm{~min}$ with TTBS buffer. The resulting immunoblots were detected using Amersham ECL Plus (GE Healthcare).

2.8. Senescence Associated Beta-Galactosidase (SA-Beta-Gal) Staining. Cells were cultured on 6-well plates at a density allowing reaching 20-30\% confluence and exposed for $24 \mathrm{~h}$ to 50 and $100 \mu \mathrm{M}$ sirtinol. After exposure, the cells were washed three times with inhibitor-free medium and cultured for up to additional 8 days. On the ninth day, the cells were fixed with $2 \%(\mathrm{v} / \mathrm{v})$ formaldehyde $/ 0.2 \%(\mathrm{v} / \mathrm{v})$ glutaraldehyde for $10 \mathrm{~min}$. The cells were then washed twice with PBS and incubated with staining solution $(30 \mathrm{mM}$ citric acid/phosphate buffer (pH 6), $5 \mathrm{mM} \mathrm{K}_{4} \mathrm{Fe}(\mathrm{CN})_{6}, 5 \mathrm{mM} \mathrm{K}_{3} \mathrm{Fe}(\mathrm{CN})_{6}, 150 \mathrm{mMNaCl}$, $2 \mathrm{mM} \mathrm{MgCl}_{2}$, and $1 \mathrm{mg} / \mathrm{mL} \mathrm{X-Gal} \mathrm{solution} \mathrm{(all} \mathrm{reagents} \mathrm{were}$ purchased from Sigma, Milan, Italy)) at $37^{\circ} \mathrm{C}$ for $24 \mathrm{~h}$. The cells were photographed and quantified with an inverted microscope (Leica, Heidelberg, Germany).

2.9. Cell Proliferation. Cells were plated $\left(5 \times 10^{3}\right.$ cells/well in 96 well plates) in RPMI 1640 (Euroclone SPA, Italy) and allowed to attach overnight. The day after, hMSCs were treated with $100 \mu \mathrm{M}$ sirtinol for 24,48 , and $72 \mathrm{~h}$. The number of living cells was measured by determination of ATP cellular levels using ViaLight Plus Kit (Lonza Group Ltd). The kit is based upon the bioluminescent measurement of ATP that is present in all metabolically active cells. The bioluminescent method utilizes an enzyme, luciferase, which catalyzes the formation of light from ATP and luciferin. The emitted light intensity is linearly related to the ATP concentration and is measured using a luminometer. RLUs (relative light units) are internal unit of the kit, proportional to the amount of light produced for ATP unit.

All experiments were performed in triplicates.

2.10. Flow Cytometry Analysis. Cells were treated with $100 \mu \mathrm{M}$ sirtinol for 24,48 , and $72 \mathrm{~h}$. Cells were resuspended in the staining solution containing RNAseA, propidium iodide $(50 \mu \mathrm{g} / \mathrm{mL})$, sodium citrate $(0.1 \%)$, and NP40 $(0.1 \%)$ in PBS $1 \mathrm{X}$ for $30 \mathrm{~min}$ in the dark. Cell cycle distribution was assessed with a FACScalibur flow cytometer (Becton Dickinson, San Jose, CA, USA), and 10.000 cells were analyzed by ModFit version 3 Technology (Verity Software House, Topsham, ME, USA) and Cell Quest (Becton Dickinson, San Jose, CA, USA) [27].

2.11. Migration Assay. Cells were plated on 24-well plates at a density allowing reaching $50-75 \%$ confluence and hMSCs were treated for 24,48 , and $72 \mathrm{~h}$ with $100 \mu \mathrm{M}$ sirtinol. A total of $1.5 \times 10^{4}$ cells were resuspended in $250 \mu \mathrm{L}$ of RPMI 1640 containing $0.2 \%$ FBS and pipetted in the upper chamber of a modified Boyden chamber (Costar Transwell assay, $8 \mu \mathrm{m}$ pore size, Corning, NY). The chamber was placed in a 24 well culture dish containing $750 \mu \mathrm{L}$ complete RPMI 1640 with $10 \%$ FBS and growth factors. After $24 \mathrm{~h}$ incubation at $37^{\circ} \mathrm{C}$, transmigrated cells were counted by independent investigators at the inverted microscope.

2.12. Capillary Tube Formation Assay in Matrigel. Cells were plated on 6-well plates at a density allowing reaching 50$75 \%$ confluence and hMSCs were treated for $24 \mathrm{~h}$ with 50 and $100 \mu \mathrm{M}$ sirtinol. For analysis of capillary tube formation, $150 \mu \mathrm{L}$ Matrigel (Becton Dickinson, San Jose, CA, USA) was laid into a 96-well plates (BD Falcon, Heidelberg, Germany) and incubated at $37^{\circ} \mathrm{C}$ for 30 minutes. Cells were trypsinized and $3 \times 10^{4}$ cells were suspended in $150 \mu \mathrm{L}$ of medium and plated onto Matrigel. Cells were incubated at $37^{\circ} \mathrm{C}$ and capillary tube formation in Matrigel was observed under an inverted microscope (Leica, Heidelberg, Germany) after 4 and $24 \mathrm{~h}$ of incubation.

2.13. Statistical Analysis. All data are represented as mean \pm S.D. Statistical significance was evaluated by performing Student's $t$-test and significance was accepted if $P$ value was $<0.05$.

\section{Results}

3.1. Immortalization of hMSCs. The ectopic expression of hTERT has been reported to extend the life span of cells [28]. 
However, the use of hTERT alone is not sufficient to immortalize hMSCs, requiring the combinatorial expression of human papillomavirus type 16 genes (HPV16) E6 and E7 [29].

Therefore, primary hMSCs were infected with HPV16 E6E7 and hTERT lentiviral vectors expressing pSin hTERT and pSin E6-E7 [30] using a multi-infection program, as reported in Methods section. Two clones were obtained and tested for the presence of hTERT and E6-E7 transcripts. Based on RT-PCR data, both clones (MeBM1E1, MeBM1E2) showed similar levels of hTERT and E6-E7 transcripts. No hTERT and E6-E7 expression were detected in untransduced hMSCs (Supplementary Figure 1(a); see the Supplementary Material available online at http://dx.doi.org/10.1155/2014/783459). The resulting cell lines maintained a fibroblast-like phenotype comparable to primary hMSCs and showed no differences in hMSCs markers expression, such as CD73, CD90, and CD105 (Supplementary Figure 1(b)). Thus, these immortalized mesenchymal cells (MeBM1E1, MeBM1E2) represent a valuable model that can be used for basic studies of mesenchymal biology.

3.2. Differential Sirtuin Expression in hMSCs. Using the MeBM1E1 clone, we assessed the expression profile of the Sirt1-Sirt7 genes. We collected RNAs from cells exposed to either $21 \% \mathrm{O}_{2}$ or $1 \% \mathrm{O}_{2}$ for $24 \mathrm{~h}$ and we performed RT-qPCR analysis to quantify their expression. As shown in Figure 1(a), a significant induction of Sirtl and Sirt7 $(P$ value $\leq 0.05)$ was detected at mRNA levels in hMSCs under hypoxic conditions compared to normoxia. No significant differences in other sirtuin transcripts were observed under the same conditions.

Next, we evaluated the contribution of hypoxia on SIRT1 protein expression and we collected lysates from cells exposed to $1 \% \mathrm{O}_{2}$ for $24 \mathrm{~h}$ in the presence or absence of growth factors (i.e., serum).

As shown in Figure 1(b), Western blot analysis showed that there was no change in SIRT1 protein levels in hMSCs exposed to $1 \% \mathrm{O}_{2}$ for $24 \mathrm{~h}$ grown in presence of serum, whereas hypoxia increased SIRT1 levels when hMSCs were cultured in low serum conditions. These data showed that hypoxia alone did not stimulate SIRT1 protein accumulation, whereas depletion of growth factors in combination with hypoxia resulted in an increase of SIRT1 protein expression.

3.3. Inhibition of SIRT1 Induces Premature Senescence-Like Phenotype in hMSCs. To evaluate the effects of targeting SIRT1, we decided to use pharmacological and genetic approaches to inhibit SIRT1. Genetic inhibition was obtained by silencing SIRT1 with lentiviral vector expressing sh-SirtlGFP. In order to determine the infection efficiency, green fluorescent protein (GFP) was monitored using fluorescence microscopy after 10 days from infection. As shown in Supplementary Figure 2(a), high levels of GFP in sh-Sirtl-hMSCs were observed with a concomitant reduction of SIRT1 protein (Supplementary Figure 2(b)). In addition, pharmacological inhibition was obtained by sirtinol, a cell permeable specific inhibitor of SIRT deacetylase activity [19].

To investigate whether SIRT1 modulates premature senescence-like phenotype in hMSCs, we examined the effect of SIRT1 inhibition in our cells. hMSCs were treated with sirtinol at 50 and $100 \mu \mathrm{M}$ for $24 \mathrm{~h}$. After exposure, the cells were washed with inhibitor-free medium and cultured for additional 8 days. As shown in Figure 1(c), sirtinol induced senescence-like morphological changes in hMSCs that showed enlarged and flattened shapes with a concomitant cell number reduction. Then, we evaluated SA- $\beta$-gal activity, a characteristic feature of senescence phenotype. Sirtinol increased SA- $\beta$-gal activity in hMSCs compared to control. Importantly, sirtinol increased SA- $\beta$-gal activity in a dose-dependent manner (Figures 1(d)-1(e)). To confirm the prosenescence role of SIRT1 inhibition, we examined SA$\beta$-gal activity in sh-Sirtl infected cells. As expected, similar results were obtained in sh-Sirtl cells (data not shown). These results demonstrated that inhibition of SIRT1 induced a senescence phenotype in hMSCs.

3.4. Effects of SIRT1 Inhibition on Proliferation. We then examined the effects of SIRT1 inhibition on the proliferation of hMSCs. Cells were treated as previously described and proliferation was measured by intracellular levels of ATP at different times $(24,48$, and $72 \mathrm{~h})$. Sirtinol significantly inhibited the proliferation of hMSCs in a time-dependent manner compared to control. Similar results were obtained in sh-Sirt1 hMSCs (Figures 2(a)-2(b)).

To investigate whether inhibition of SIRT1 induced growth arrest or cell death, a cell cycle analysis was performed on hMSCs treated with sirtinol for 24,48 , and $72 \mathrm{~h}$ by fluorescence activated cell sorting (FACS). By this analysis, we observed a significant cell accumulation in pre-G1 phase, corresponding to apoptotic cells after 24,48 , and $72 \mathrm{~h}$ of treatment compared to untreated cells. No significant effect was observed in G1, G2, and S phase (Figure 2(c)), compared to control. Similar results were obtained in sh-Sirtl cells (data not shown). These data suggested that inhibition of SIRT1, obtained by both pharmacological and genetic approaches, induced apoptosis in hMSCs, without altering the cell cycle distribution of the cells.

3.5. SIRT1 Inhibition Impairs Migration and Capillary Tube Network Formation. Then, we examined whether inhibition of SIRT1 impinged on the migration ability of hMSCs. Cells were exposed for 24,48 , and $72 \mathrm{~h}$ to $100 \mu \mathrm{M}$ sirtinol and we examined the migratory ability of hMSCs in presence of growth factors. The number of migrated cells was significantly reduced in cells treated with SIRT1 inhibitor in a time-dependent manner (Figure 3(a)), compared to control. Consistent with these findings, silenced SIRT1 resulted in a significant reduction of migration compared to control (Figure 3(b)).

These results suggested that inhibition of SIRT1 reduces the capability of hMSCs to migrate. To further assess whether inhibition of SIRT1 might play a role in the ability to form capillary-like networks, we performed a tubule formation assay. We used Matrigel as the basement matrix to induce tubule formation. Cells were exposed to sirtinol (50 and $100 \mu \mathrm{M}$ ) for $24 \mathrm{~h}$; then sirtinol was removed from the culture media. The cells were plated on Matrigel and allowed to form 


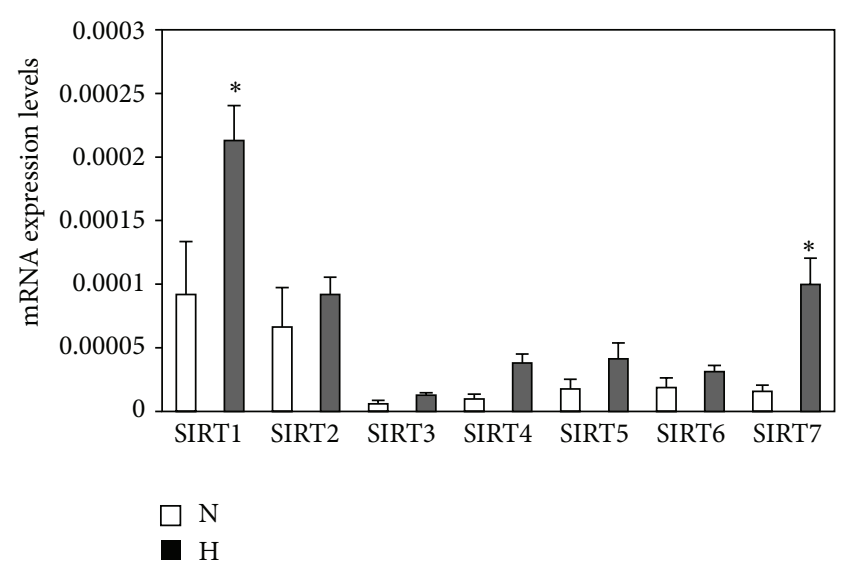

(a)

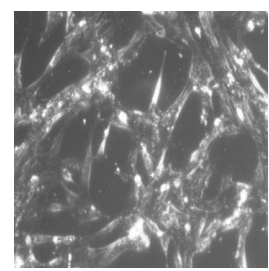

$\underline{50 \mu \mathrm{m}}$ CTR

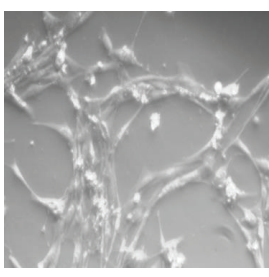

$50 \mu \mathrm{M}$ sirtinol

(c)

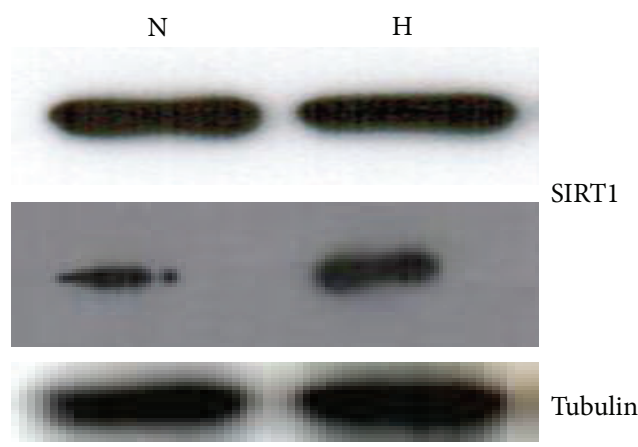

(b)

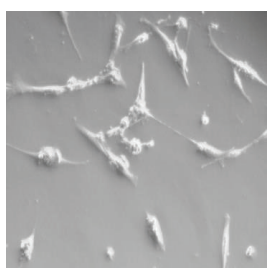

$100 \mu \mathrm{M}$ sirtinol

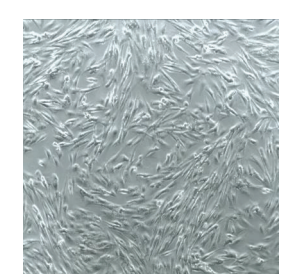

$\underline{200 \mu \mathrm{m} \quad \text { CTR }}$

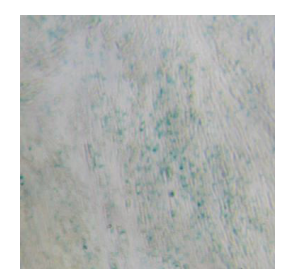

$50 \mu \mathrm{M}$ sirtinol

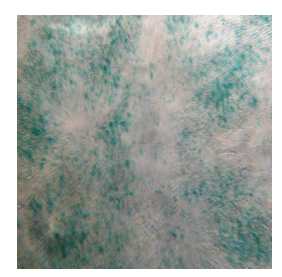

$100 \mu \mathrm{M}$ sirtinol

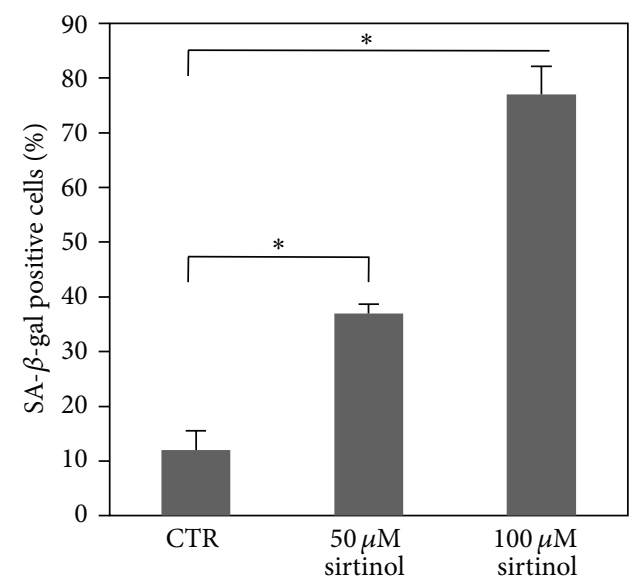

(d)

(e)

FIGURE 1: SIRT1-SIRT7 expression in hMSCs and effects of SIRT1 inhibition on phenotype. (a) SIRT1-SIRT7 mRNA levels were measured by real-time PCR (RT-PCR) analyses of total RNAs obtained from hMSCs exposed to either $21 \% \mathrm{O}_{2}(\mathrm{~N})$ or $1 \% \mathrm{O}_{2}(\mathrm{H})$ for $24 \mathrm{~h}$ and relative expression $\left( \pm S D\right.$ ) were shown. (b) Western blot analysis of hMSCs grown at either $21 \% \mathrm{O}_{2}(\mathrm{~N})$ or $1 \% \mathrm{O}_{2}(\mathrm{H})$ for 24 h under normal conditions (upper panel) and serum-starved (lower panel). Antibodies against SIRT1 and tubulin were used. (c) Morphological changes in hMSCs were examined 8 days after treatment with sirtinol $(50$ and $100 \mu \mathrm{M})$ for $24 \mathrm{~h}(40 \mathrm{x}$ magnification, scale bars $=50 \mu \mathrm{m})$. (d) Representative photographs of blue-stained cells for SA- $\beta$-Gal activity are shown (10x magnification, scale bars $=200 \mu \mathrm{m})$ at 8 days after sirtinol $(50$ and $100 \mu \mathrm{M})$ treatment compared to control (CTR). (e) SA- $\beta$-Gal-positive cells were quantified by counting in at least 3 random fields for each condition. Results show the mean of three independent experiments. Graph represents means $\pm \mathrm{SD}, n=3$. * indicates statistical significance from control, $P \leq 0.05$.

tubule networks in vitro. As shown in Figure 3(c), we found that hMSCs exposed to $50 \mu \mathrm{M}$ sirtinol formed less developed tubule structures than untreated cells within a 4-hour period and the number of branch points significantly diminished (Figure 3(d)). Additionally, cell treatment at a concentration of $100 \mu \mathrm{M}$ sirtinol resulted in the complete suppression of tubule-like structure formation, in contrast to stable tubular networks present in the control (Figure 3(c), upper panel$3 \mathrm{D})$. A reduced ability to form tubule-like formation was also observed in sh-Sirtl cells compared to control (Figure 3(c) lower panel-3D). These data indicated that SIRT1 activity is involved in tubule-like formation of hMSCs. 


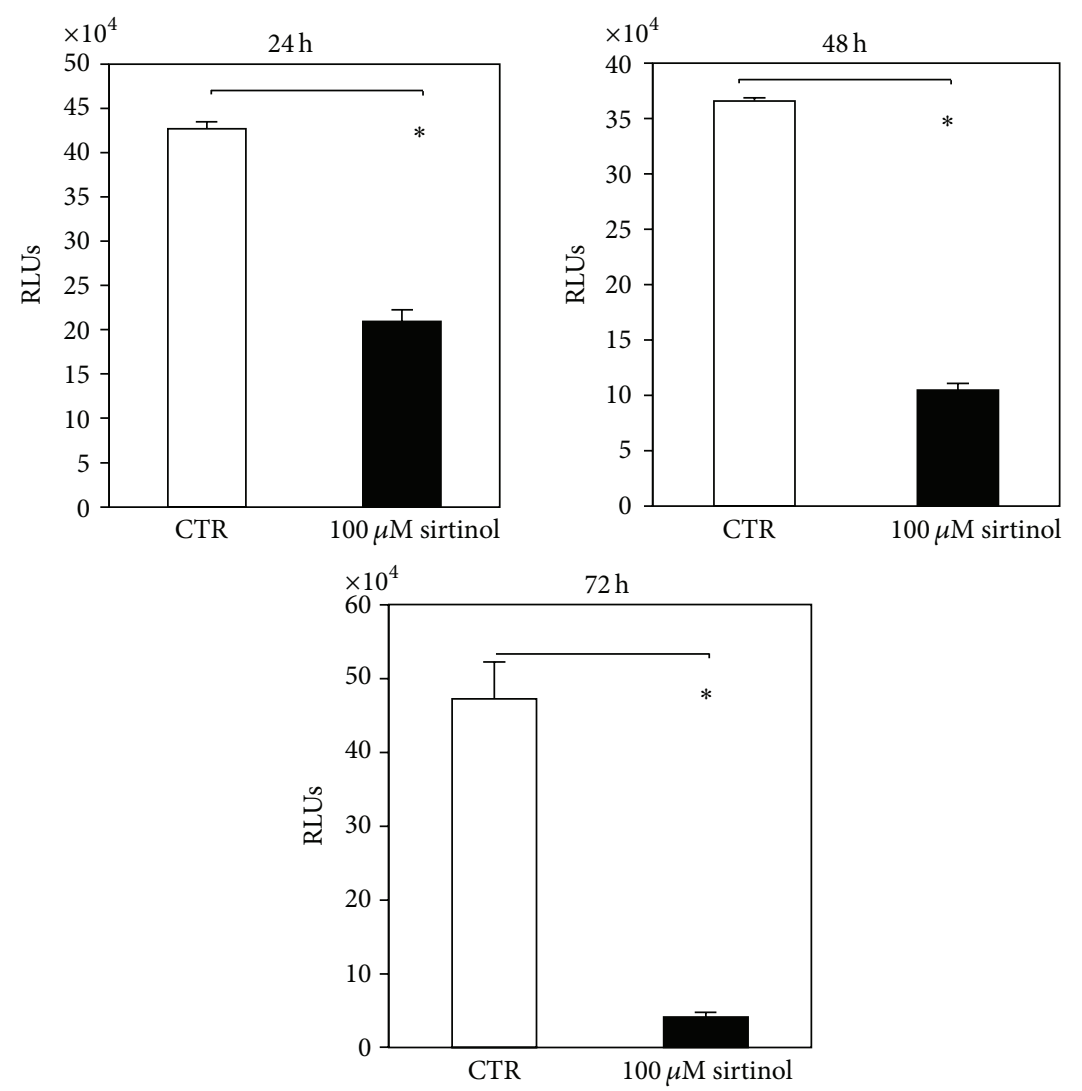

(a)
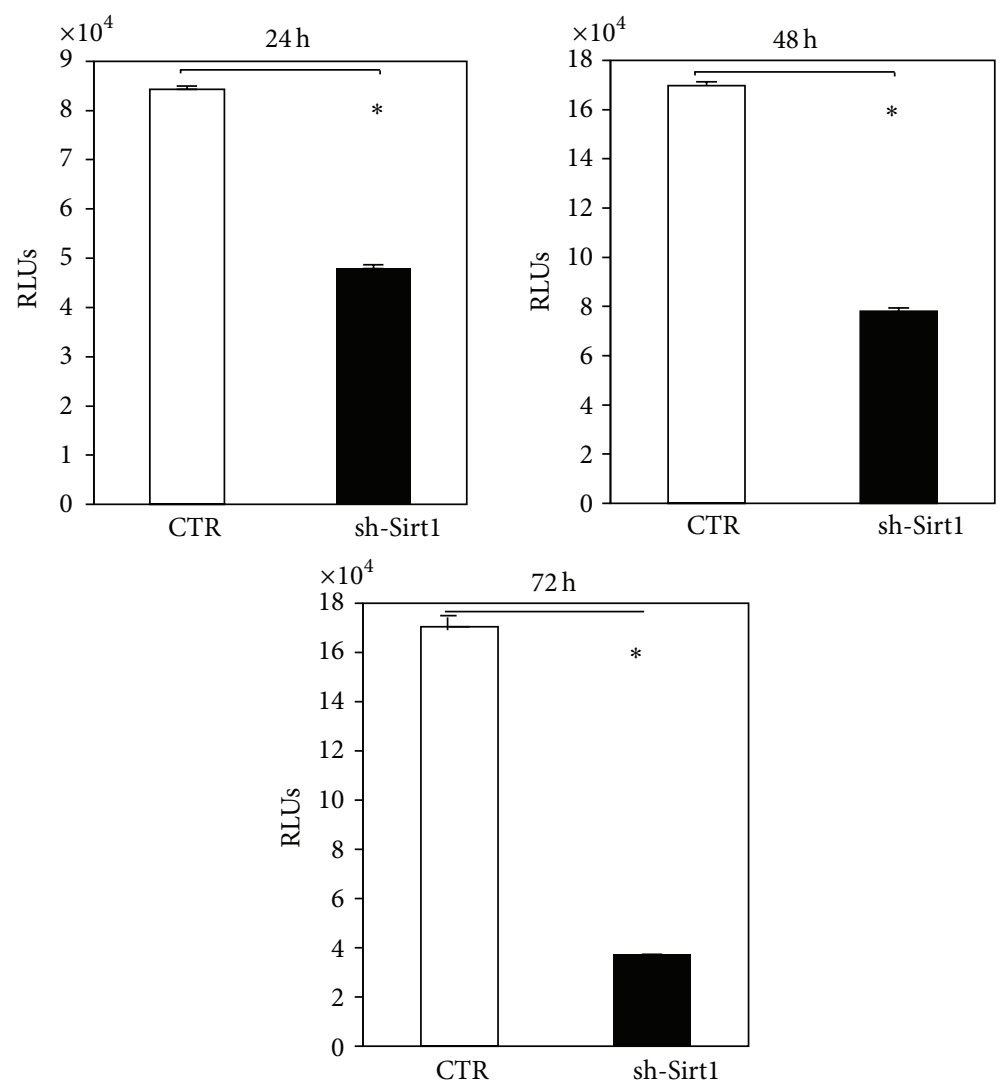

(b)

FIGURE 2: Continued. 


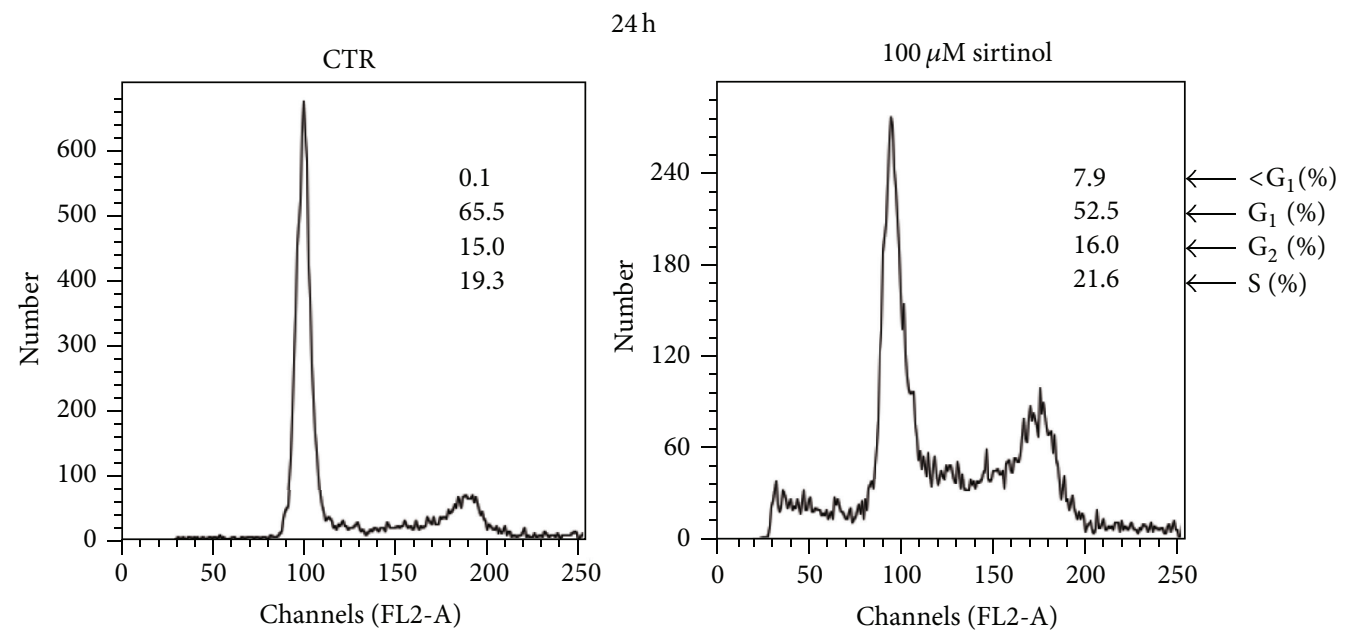

$48 \mathrm{~h}$
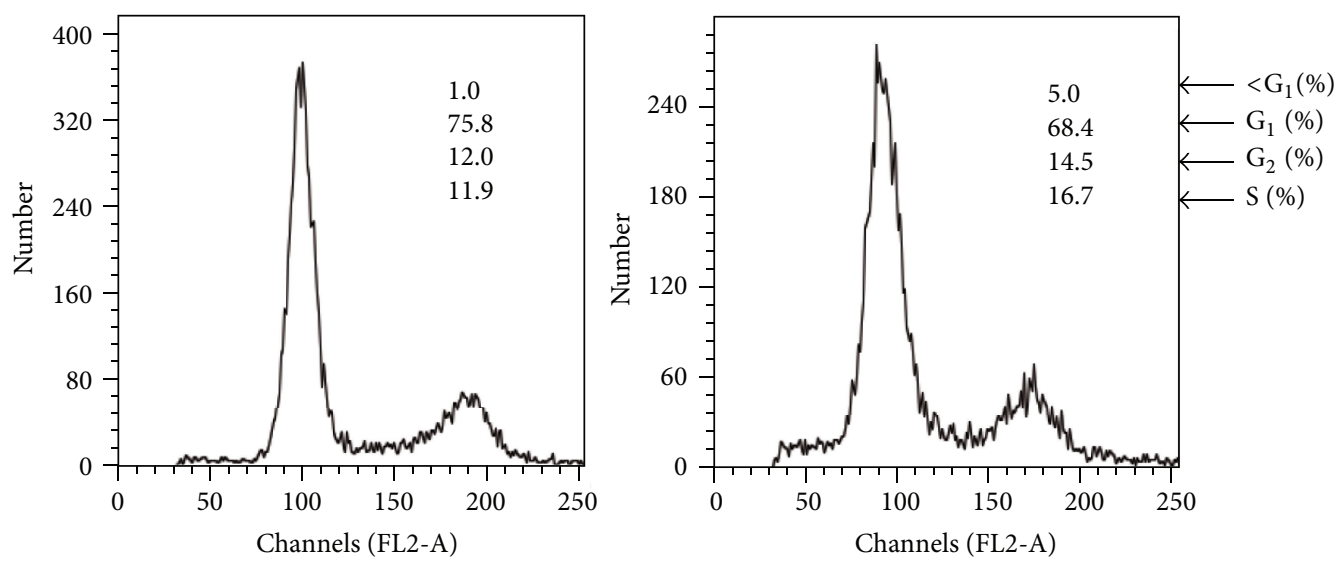

$72 \mathrm{~h}$
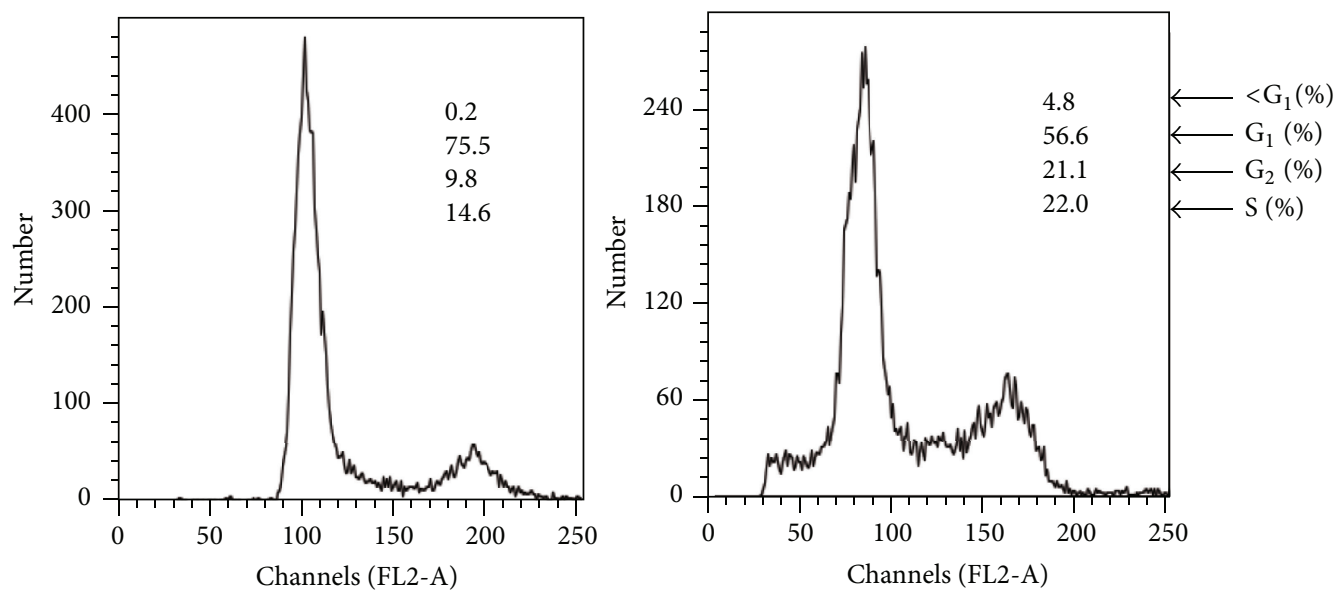

(c)

FIGURE 2: Effects of SIRT1 inhibition on proliferation, cell cycle, and apoptosis. (a) hMSCs were treated with sirtinol $100 \mu \mathrm{M}$ (black bars) or equivalent concentration of DMSO (white bars) for 24,48 , and $72 \mathrm{~h}$. (b) sh-Sirtl infected hMSCs were plated and proliferation was measured for 24, 48, and $72 \mathrm{~h}$. Each histogram indicates the RLUs ${ }^{\#}$ related to cell growth measured at different times. Error bars represent SD of $n=3$. * denotes statistical differences when $P \leq 0.05$ is compared to control. (c) hMSCs were treated with sirtinol $100 \mu \mathrm{M}$ or equivalent concentration of DMSO for 24,48 , and $72 \mathrm{~h}$. The cells stained with propidium iodide (PI) were subjected to flow cytometric analysis to determine the cell distributions at each phase of the cell cycle. ${ }^{\#}$ RLUs are relative light units (RLUs) related to ATP cellular level. 

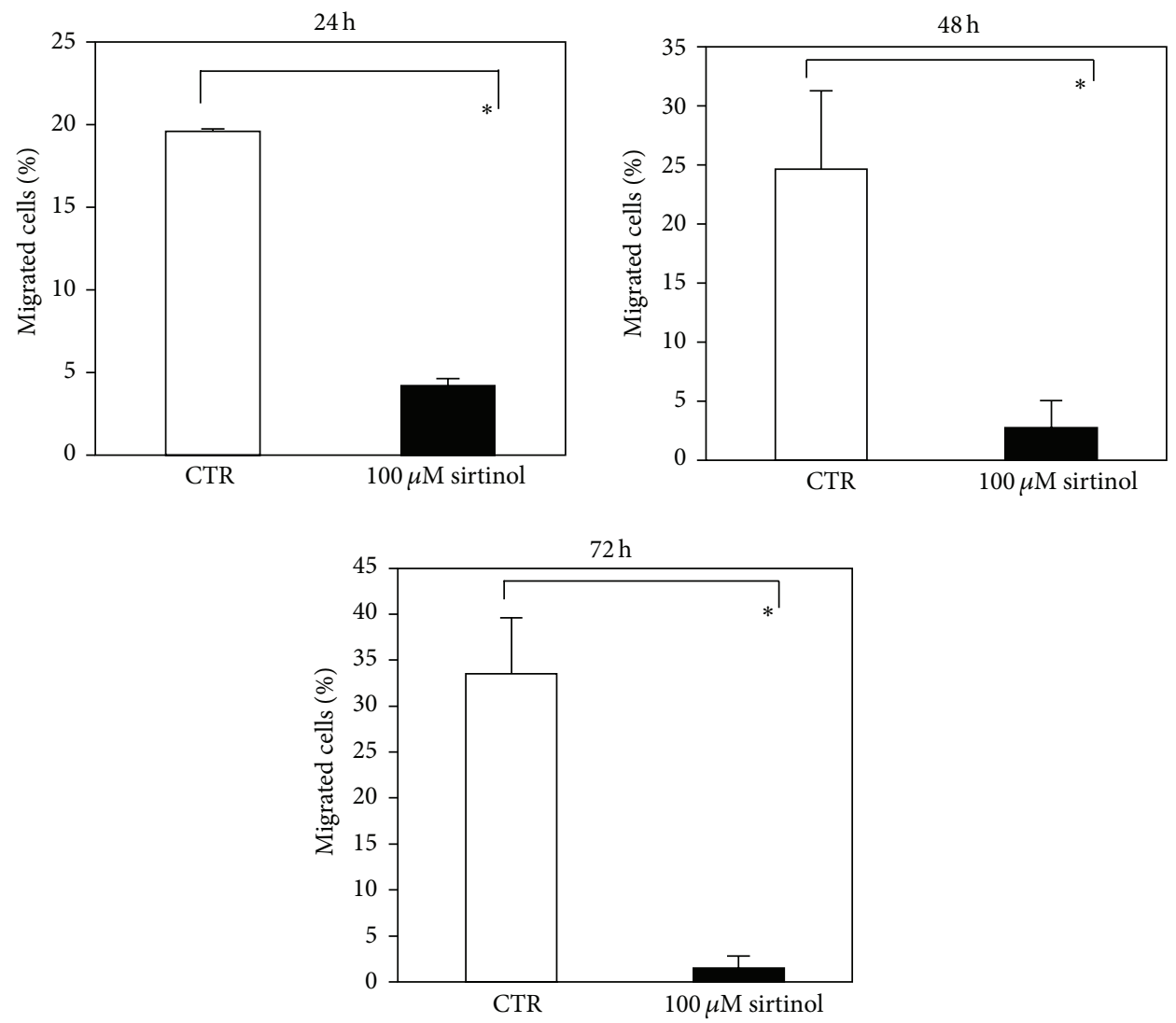

(a)
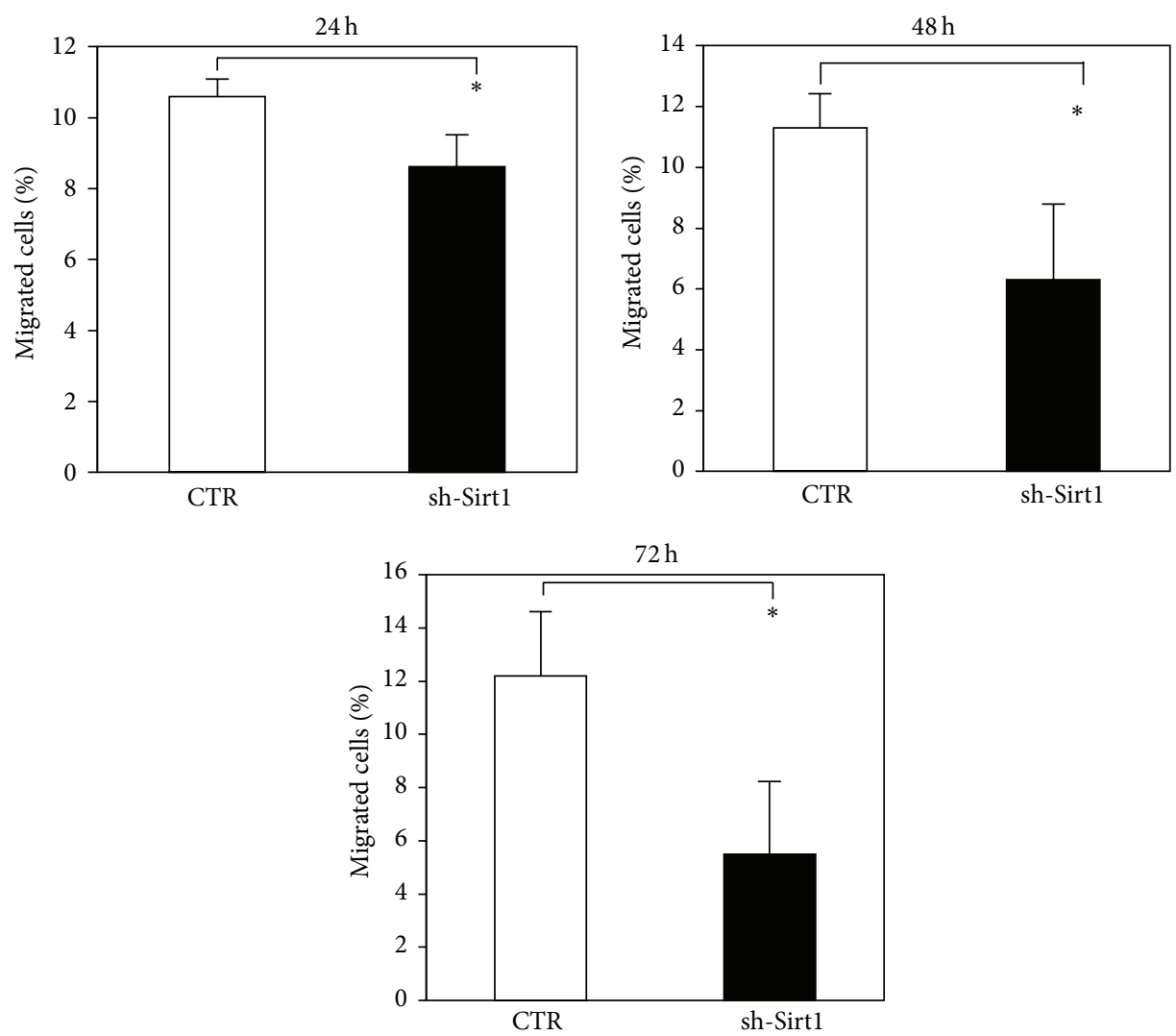

(b)

FIgure 3: Continued. 


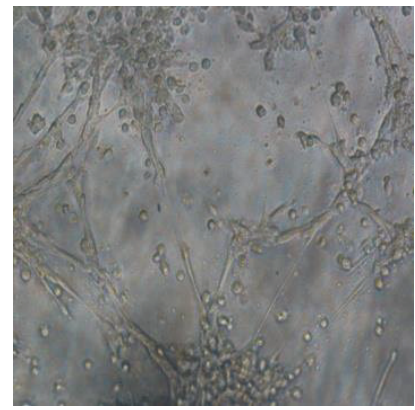

$50 \mu \mathrm{M}$ sirtinol

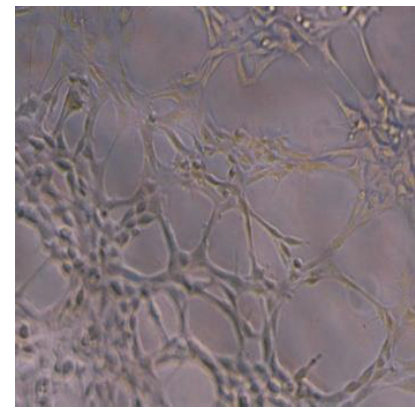

$2 \underline{00 \mu \mathrm{m}}$

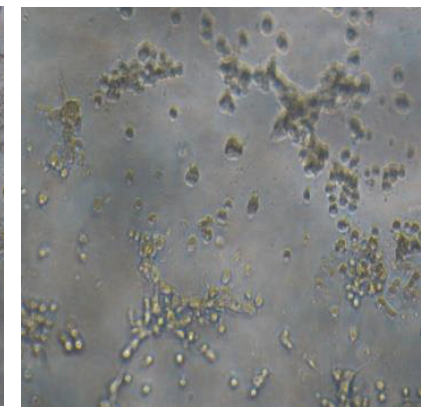

$100 \mu \mathrm{M}$ sirtinol

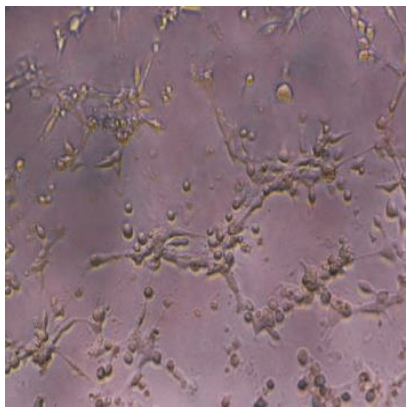

sh-Sirt1

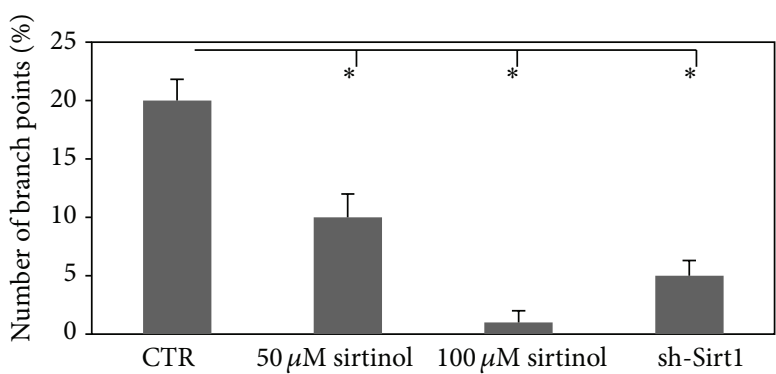

(c)

(d)

FIGURE 3: Effects of SIRT1 inhibition on migration and capillary tube network formation. (a) hMSCs were treated with sirtinol $100 \mu \mathrm{M}$ (black bars) or equivalent concentration of DMSO (white bars) for 24, 48, and $72 \mathrm{~h}$. (b) sh-Sirtl-hMSCs migration (black bars) compared to control (white bars) at 24, 48, and $72 \mathrm{~h}$. Each histogram indicates the $\%$ of migrated cells measured at different times. Statistical differences were denoted with $*$ when $P \leq 0.05$ is compared to control. (c) Tubule formation promoted by hMSCs treated with sirtinol (50 and $100 \mu \mathrm{M})$ (upper panel) and tubular structures of sh-Sirtl infected hMSCs plated on Matrigel compared to control (lower panel). Magnification 10x. Scale bar $=200 \mu \mathrm{m}$. (d) Tubule formation was quantified by counting the number of branch points of the capillary network. Data are expressed as mean \pm SD. $*$ denotes statistical differences when $P \leq 0.05$ is compared to control.

3.6. Impaired Angiogenic Properties of hMSCs Resulting from SIRT1 Inhibition Are Mediated by HIF-1 $\alpha$ Protein. To investigate whether SIRT1 activity could regulate HIF-1 $\alpha$ accumulation, we analyzed the effects of SIRT1 inhibition on HIF-1 $\alpha$.

Cells were incubated with $100 \mu \mathrm{M}$ sirtinol for $24 \mathrm{~h}$ and then exposed to $1 \% \mathrm{O}_{2}$ for $6 \mathrm{~h}$, known to induce HIF-1 $\alpha$. We then collected cell lysates and Western blotting analysis was performed. Interestingly, as shown in Figure 4(a), inhibition of SIRT1 led to a reduction of SIRT1 protein, as expected, and a strong repression of HIF-1 $\alpha$ protein accumulation in hypoxic conditions, compared to control.

To determine if reduction in HIF- $1 \alpha$ levels, conferred by SIRT1 inhibition, could affect HIF- $1 \alpha$ transcriptional activity, we looked at the expression of known HIF-1 $\alpha$ target genes. Cells were treated with $100 \mu \mathrm{M}$ sirtinol for $24 \mathrm{~h}$ and exposed to either $21 \% \mathrm{O}_{2}$ or $1 \% \mathrm{O}_{2}$ for $6 \mathrm{~h}$. Then, we collected RNAs from cells and RT-qPCR analysis was performed. As shown in Figure 4(b), the treatment with sirtinol in combination with hypoxia significantly reduced the hypoxic induction of SIRT1 expression and HIF- $1 \alpha$ target genes: Glutl and VEGF. These data indicated that inhibition of SIRT1 decreased HIF$1 \alpha$ protein accumulation and its transcriptional activity under hypoxic conditions.

\section{Discussion}

In the present study, we have generated an immortalized human bone marrow-derived mesenchymal cell line (MeBM) that represent a valuable tool to study mesenchymal biology and we investigated whether SIRT1 can influence angiogenic capacity of these cells.

Cell therapy with hMSCs is a promising and safe modality with the potential for vascular regeneration in the treatment of several diseases, especially critical limb ischemia. Pilot human studies have shown that autologous transplantation of bone marrow cells induced therapeutic angiogenesis in patients with critical limb ischemia [3]. hMSCs are pluripotent progenitors that can differentiate into a variety of cell types and have been shown to promote angiogenesis both in vivo and in vitro. It is widely accepted that hMSCs present in bone marrow are therapeutic cells. Emerging evidence suggests that most of the beneficial effects of hMSCs can be explained by the secretion of soluble factors that induce endogenous reparatory processes. However, the mechanisms by which hMSCs promote angiogenesis are not clear.

The present investigation was undertaken to identify the function, if any, of SIRT1 in hMSCs and clarify the molecular 


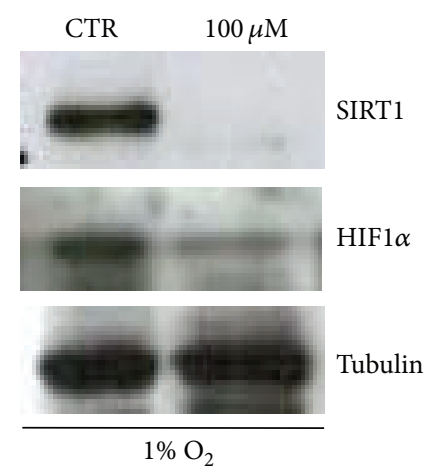

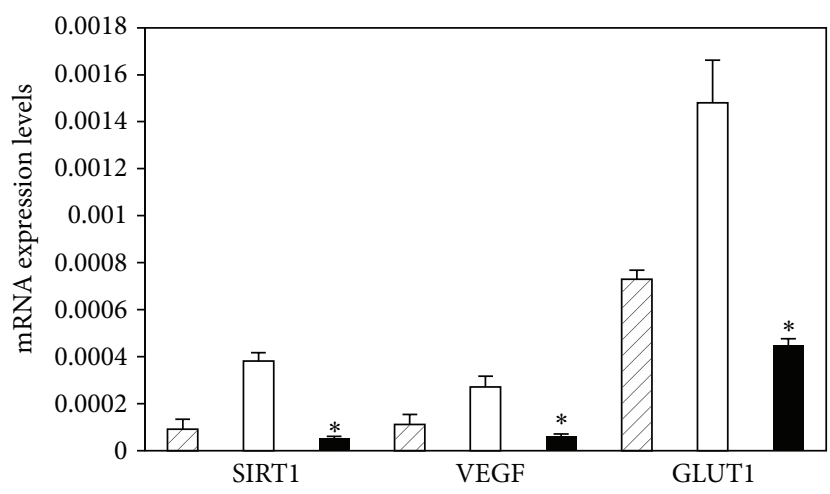

$\square \mathrm{N}$
$\square \mathrm{H}$
$\mathrm{H}+$ sirtinol

(a)

(b)

FIGURE 4: Effects of sirtinol on SIRT1 expression and HIF- $1 \alpha$. (a) hMSCs were treated with sirtinol (100 $\mu \mathrm{M})$ or equivalent concentration of DMSO (CTR) for $24 \mathrm{~h}$ and then exposed to $1 \% \mathrm{O}_{2}$ for $6 \mathrm{~h}$. All cells lysates were analyzed for SIRT1, HIF- $1 \alpha$, and tubulin by Western blot. (b) The amounts of SIRT1, VEGF, and GLUT1 mRNA of hMSCs treated with sirtinol at concentration of $100 \mu \mathrm{M}$ (black bars) or equivalent concentration of DMSO (white bars) for $24 \mathrm{~h}$ and exposed to $1 \% \mathrm{O}_{2}(\mathrm{H})$ for $6 \mathrm{~h}$ were quantified with real-time PCR. The levels of SIRT1, VEGF, and GLUT1 mRNA of hMSCs exposed to $21 \% \mathrm{O}_{2}(\mathrm{~N})$ for $24 \mathrm{~h}$ were measured. * indicates statistical significance from control, $P \leq 0.05$.

mechanism by which SIRT1 regulate angiogenesis in hypoxic conditions.

Sirtuins represent a family of NAD+-dependent protein deacetylases involved in several pathologies. In mammals, SIRT1 is the most closely related homologue of yeast Sir 2 and belongs to class III histone deacetylases. It regulates a wide variety of biological functions including gene expression, cell survival, proliferation, differentiation, metabolism, immune response, carcinogenesis, and angiogenic response through multiple targets [15].

Investigation of sirtuin expression was an important preliminary step to study the selective role of class III HDACs. Since sirtuins have been shown to respond to perturbations in the ratio of oxidized $\mathrm{NAD}+/$ reduced $\mathrm{NADH}$ and, therefore, to modulate the response to hypoxic stress [31], we first looked at sirtuin expression of the family members, both in normoxic and hypoxic conditions.

Therefore, we assessed the expression profile of the Sirtl7 genes in our cells. We found that SIRT1 and SIRT7 were significantly upregulated in hypoxic conditions compared to normoxia.

SIRT1 activation improves endothelial function and suppresses vascular inflammation, two central pathophysiological processes involved in the initiation and progression of cardiovascular disease [32]. In particular, SIRT1 has been shown to protect endothelial cells from premature senescence and to regulate angiogenesis and vascular tone [26]; thus we decided to focus our study on SIRT1.

In our study, we evaluated the contribution of hypoxia on SIRT1 protein, showing that no change in SIRT1 was detected in hMSCs grown in presence of growth factors, whereas SIRT1 protein expression increased in hMSCs grown in absence of growth factors under hypoxic conditions. Other experiments will be necessary to clarify this; however, our findings might indicate that SIRT1 may act as a sensor of the metabolic state of MSCs in stress conditions (i.e., hypoxia and absence of growth factors).

Several studies have demonstrated that SIRT1 prevents the onset of senescence in multiple cell types, which exhibit alterations in morphology and gene expression that extinguish essential cellular functions [31].

It is known that primary cells underwent senescence when cultured in vitro, showing increased activity of $\beta$ galactosidase ( $\beta$-gal) when assayed at $\mathrm{pH} 6$ [22]. Therefore, we decided to evaluate whether inhibition of SIRT1 might play a role in undertaking a senescent phenotype in our cells. We found that sirtinol, a cell-permeable 2-hydroxy-1napthaldehyde derivative that acts as a specific and direct inhibitor of all NAD+-dependent protein deacetylases of sirtuin family, significantly increased SA- $\beta$-gal activity in a concentration-dependent manner and sustained enlarged and flattened cell morphology.

Our results are consistent with previous studies in human endothelial cells [22, 33], mouse fibroblasts [34], and human cancer cells [35], concluding that sirtuins are implicated in cellular senescence.

It has been demonstrated that senescence alters mesenchymal stem cell properties, such as proliferation and migration [36] and activity of SIRT1 has been linked to this condition [37]. In fact, when cells undergo senescence, their proliferation declines significantly.

Our findings showed that SIRT1 inhibition significantly inhibited the proliferation rate of hMSCs in a time-dependent manner and significantly induced apoptosis in hMSCs.

Going through our data, we also noticed that the effect of genetic inhibition of SIRT1 on angiogenesis response of 
hMSCs was less pronounced than pharmacological modulation of SIRT1. A possible explanation for this observation is that sirtinol can inhibit other NAD+-dependent protein deacetylases of sirtuin family, especially SIRT2 [22]. In conclusion, we suggested that inhibition of SIRT1 both by pharmacological and genetic approaches reduces significantly angiogenic properties in cultured hMSCs.

The connection between sirtuin and HIF proteins is complex and the current literature is in part contradictory [19]. Recent reports have linked HIF to sirtuin families by demonstrating that SIRT1, SIRT3, SIRT6, and SIRT7 can regulate the activity of HIF proteins $[19,38]$. Lim et al. [18] demonstrated that SIRT1 binds to and deacetylates HIF- $1 \alpha$ at lysine 674 . This interaction blocks p300 recruitment to the promoter of HIF- $1 \alpha$ target genes and thereby represses HIF$1 \alpha$ transcriptional activity, whereas Laemmle et al. reported that SIRT1 increased HIF-1 $\alpha$ protein levels [19].

In contrast, Dioum et al. [17] have reported that SIRT1 does not target HIF- $1 \alpha$; it rather deacetylates HIF- $2 \alpha$ and their interaction promotes HIF- $2 \alpha$ transcriptional activity. In addition, because SIRT1 is a redox cellular sensor and dependent on metabolic status of the cell, its regulation by hypoxia has been a point of interest. In one report, SIRT1 is downregulated in hypoxic conditions due to decreased $\mathrm{NAD}+$ levels [18], while in another study it is upregulated in a HIF-dependent manner [39].

Thus, the interaction between SIRT1 and HIF factors and the resulting outcome of their interactions are still unclear. In this study we found that HIF- $1 \alpha$ transcriptional activity is impaired by SIRT1 inhibition under hypoxic conditions, as reported in [19].

\section{Conclusions}

Our results suggest that SIRT1 exert a role in angiogenic properties of hMSCs. Thus, our study might have important implications in the field of angiogenesis, possibly leading to the identification of chemical compounds that can positively regulate SIRT1, improving regenerative processes exerted by hMSCs.

\section{Conflict of Interests}

The authors have stated that they have no conflict of interests.

\section{Acknowledgments}

The authors are grateful to Dr. Antonio Barbieri, Dr. Giosue' Scognamiglio, and Dr. Giuseppina Liguori for microscope assistance. Special thanks are due to Dr. Gabriella Malzone and Dr. Monica Cantile for important suggestions. The authors are grateful to PON01_01227 (Botti Chiara) which gave them the support needed to perform the research.

\section{References}

[1] G. Ren, X. Chen, F. Dong et al., "Concise review: mesenchymal stem cells and translational medicine: emerging issues," Stem Cells Translational Medicine, vol. 1, no. 1, pp. 51-58, 2012.
[2] C. Stamm, B. Westphal, H. D. Kleine et al., "Autologous bonemarrow stem-cell transplantation for myocardial regeneration," The Lancet, vol. 361, no. 9351, pp. 45-46, 2003.

[3] A. Schiavetta, C. Maione, C. Botti et al., "A phase II trial of autologous transplantation of bone marrow stem cells for critical limb ischemia: results of the Naples and Pietra Ligure evaluation of stem cells study," Stem Cells Translational Medicine, vol. 1, no. 7, pp. 572-578, 2012.

[4] T. Kinnaird, E. Stabile, M. S. Burnett et al., "Marrow-derived stromal cells express genes encoding a broad spectrum of arteriogenic cytokines and promote in vitro and in vivo arteriogenesis through paracrine mechanisms," Circulation Research, vol. 94, no. 5, pp. 678-685, 2004.

[5] J. Rehman, D. Traktuev, J. Li et al., "Secretion of angiogenic and antiapoptotic factors by human adipose stromal cells," Circulation, vol. 109, no. 10, pp. 1292-1298, 2004.

[6] H. M. Mi, Y. K. Sun, J. K. Yeon et al., "Human adipose tissuederived mesenchymal stem cells improve postnatal neovascularization in a mouse model of hindlimb ischemia," Cellular Physiology and Biochemistry, vol. 17, no. 5-6, pp. 279-290, 2006.

[7] R. Estrada, N. Li, H. Sarojini, J. An, M. Lee, and E. Wang, "Secretome from mesenchymal stem cells induces angiogenesis via Cyr61," Journal of Cellular Physiology, vol. 219, no. 3, pp. 563571, 2009.

[8] G. Cobellis, C. Maione, C. Botti et al., "Beneficial effects of VEGF secreted from stromal cells in supporting endothelial cell functions: therapeutic implications for critical limb ischemia," Cell Transplantation, vol. 19, no. 11, pp. 1425-1437, 2010.

[9] R. H. Lee, J. Y. Oh, H. Choi, and N. Bazhanov, "Therapeutic factors secreted by mesenchymal stromal cells and tissue repair," Journal of Cellular Biochemistry, vol. 112, no. 11, pp. 3073-3078, 2011.

[10] P. Simic, K. Zainabadi, E. Bell et al., "SIRT1 regulates differentiation of mesenchymal stem cells by deacetylating $\beta$-catenin," EMBO Molecular Medicine, vol. 5, no. 3, pp. 430-440, 2013.

[11] A. Z. Herskovits and L. Guarente, "Sirtuin deacetylases in neurodegenerative diseases of aging," Cell Research, vol. 23, no. 6, pp. 746-758, 2013.

[12] M. C. Haigis and D. A. Sinclair, "Mammalian sirtuins: Biological insights and disease relevance," Annual Review of Pathology: Mechanisms of Disease, vol. 5, pp. 253-295, 2010.

[13] T. Finkel, C. Deng, and R. Mostoslavsky, "Recent progress in the biology and physiology of sirtuins," Nature, vol. 460, no. 7255, pp. 587-591, 2009.

[14] L. Guarente, "Sirtuins, aging, and medicine," The New England Journal of Medicine, vol. 364, no. 23, pp. 2235-2244, 2011.

[15] V. Carafa, A. Nebbioso, and L. Altucci, "Sirtuins and disease: the road ahead," Frontiers in Pharmacology, vol. 3, article 4, Article ID Article 4, 2012.

[16] G. Corbi, A. Bianco, V. Turchiarelli et al., "Potential mechanisms linking atherosclerosis and increased cardiovascular risk in COPD: focus on sirtuins," International Journal of Molecular Sciences, vol. 14, no. 6, pp. 12696-12713, 2013.

[17] E. M. Dioum, R. Chen, M. S. Alexander et al., "Regulation of hypoxia-inducible factor 2alpha signaling by the stressresponsive deacetylase sirtuin 1," Science, vol. 324, no. 5932, pp. 1289-1293, 2009.

[18] J. H. Lim, Y. M. Lee, Y. S. Chun, J. Chen, J. Kim, and J. Park, "Sirtuin 1 modulates cellular responses to hypoxia by deacetylating hypoxia-inducible factor 1alpha," Molecular Cell, vol. 38 , no. 6 , pp. 864-878, 2010. 
[19] A. Laemmle, A. Lechleiter, V. Roh et al., "Inhibition of SIRT1 impairs the accumulation and transcriptional activity of HIF$1 \alpha$ protein under hypoxic conditions," PLoS ONE, vol. 7, no. 3, Article ID e33433, 2012.

[20] C. W. Pugh and P. J. Ratcliffe, "Regulation of angiogenesis by hypoxia: role of the HIF system," Nature Medicine, vol. 9, no. 6, pp. 677-684, 2003.

[21] M. Potente, L. Ghaeni, D. Baldessari et al., "SIRT1 controls endothelial angiogenic functions during vascular growth," Genes and Development, vol. 21, no. 20, pp. 2644-2658, 2007.

[22] H. Ota, M. Akishita, M. Eto, K. Iijima, M. Kaneki, and Y. Ouchi, "Sirtl modulates premature senescence-like phenotype in human endothelial cells," Journal of Molecular and Cellular Cardiology, vol. 43, no. 5, pp. 571-579, 2007.

[23] S. Portmann, R. Fahrner, A. Lechleiter et al., "Antitumor effect of SIRT1 inhibition in human HCC tumor models in vitro and in vivo," Molecular Cancer Therapeutics, vol. 12, no. 4, pp. 499508, 2013.

[24] D. Rotili, D. Tarantino, A. Nebbioso et al., "Discovery of salermide-related sirtuin inhibitors: binding mode studies and antiproliferative effects in cancer cells including cancer stem cells," Journal of Medicinal Chemistry, vol. 55, no. 24, pp. 1093710947, 2012.

[25] I. Gorenne, S. Kumar, K. Gray et al., "Vascular smooth muscle cell sirtuin 1 protects against dna damage and inhibits atherosclerosis," Circulation, vol. 127, no. 3, pp. 386-396, 2013.

[26] M. Potente, "An energy-sensor network takes center stage during endothelial aging," Circulation Research, vol. 106, no. 8, pp. 1316-1318, 2010.

[27] M. Miceli, G. Franci, C. Dell'Aversana et al., "MePR: a novel human mesenchymal progenitor model with characteristics of pluripotency," Stem Cells and Development, vol. 22, no. 17, pp. 2368-2383, 2013.

[28] A. G. Bodnar, M. Ouellette, M. Frolkis et al., "Extension of lifespan by introduction of telomerase into normal human cells," Science, vol. 279, no. 5349, pp. 349-352, 1998.

[29] T. Okamoto, T. Aoyama, T. Nakayama et al., "Clonal heterogeneity in differentiation potential of immortalized human mesenchymal stem cells," Biochemical and Biophysical Research Communications, vol. 295, no. 2, pp. 354-361, 2002.

[30] S. S. Akimov, A. Ramezani, T. S. Hawley, and R. G. Hawley, "Bypass of senescence, immortalization, and transformation of human hematopoietic progenitor cells," Stem Cells, vol. 23, no. 9, pp. 1423-1433, 2005.

[31] M. F. Oellerich and M. Potente, "FOXOs and sirtuins in vascular growth, maintenance, and aging," Circulation Research, vol. 110, no. 9, pp. 1238-1251, 2012.

[32] J. Gracia-Sancho, G. Villarreal, Y. Zhang, and G. GarcíaCardeña, "Activation of SIRT1 by resveratrol induces KLF2 expression conferring an endothelial vasoprotective phenotype," Cardiovascular Research, vol. 85, no. 3, pp. 514-519, 2010.

[33] A. Cardus, A. K. Uryga, G. Walters, and J. D. Erusalimsky, "SIRT6 protects human endothelial cells from DNA damage, telomere dysfunction, and senescence," Cardiovascular Research, vol. 97, no. 3, pp. 571-579, 2013.

[34] E. Langley, M. Pearson, M. Faretta et al., "Human SIR2 deacetylates p53 and antagonizes PML/p53-induced cellular senescence," EMBO Journal, vol. 21, no. 10, pp. 2383-2396, 2002.

[35] H. Ota, E. Tokunaga, K. Chang et al., "Sirt1 inhibitor, Sirtinol, induces senescence-like growth arrest with attenuated RasMAPK signaling in human cancer cells," Oncogene, vol. 25, no. 2, pp. 176-185, 2006.
[36] S. D. Barros, S. Dehez, E. Arnaud et al., "Aging-related decrease of human ASC angiogenic potential is reversed by hypoxia preconditioning through ROS production," Molecular Therapy, vol. 21, no. 2, pp. 399-408, 2013.

[37] N. Engel and U. Mahlknecht, "Aging and anti-aging: unexpected side effects of everyday medication through sirtuin 1 modulation," International Journal of Molecular Medicine, vol. 21, no. 2, pp. 223-232, 2008.

[38] M. E. Hubbi, H. Hu, D. M. Gilkes, and G. L. Semenza, "Sirtuin7 inhibits the activity of hypoxia-inducible factors," Journal of Biological Chemistry, vol. 288, no. 29, pp. 20768-20775, 2013.

[39] R. Chen, M. Xu, R. T. Hogg et al., "The acetylase/deacetylase couple CREB-binding protein/sirtuin 1 controls hypoxiainducible factor 2 signaling," The Journal of Biological Chemistry, vol. 287, no. 36, pp. 30800-30811, 2012. 


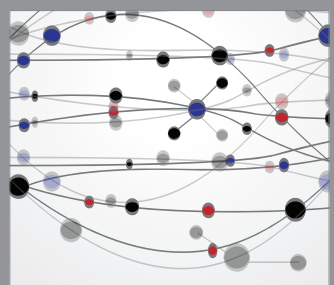

The Scientific World Journal
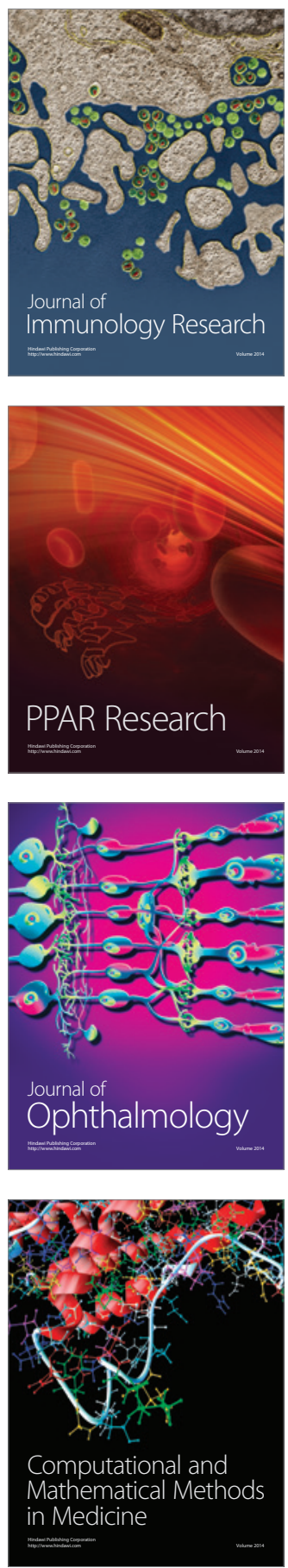

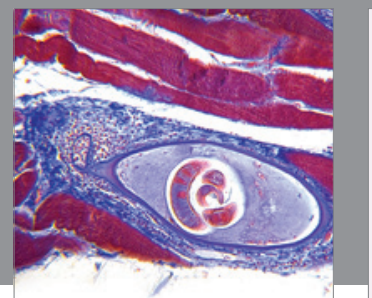

Gastroenterology

Research and Practice
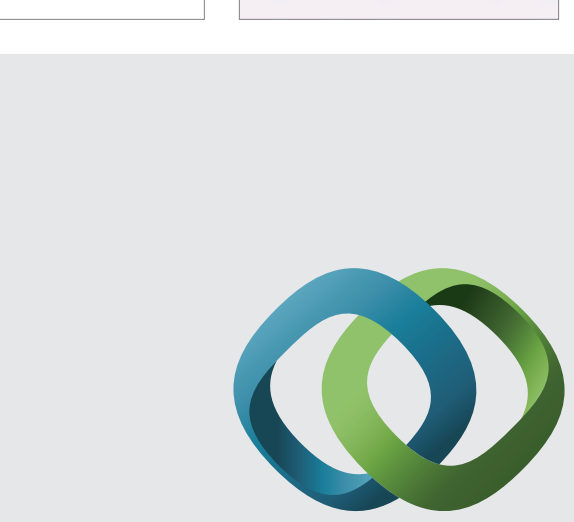

\section{Hindawi}

Submit your manuscripts at

http://www.hindawi.com
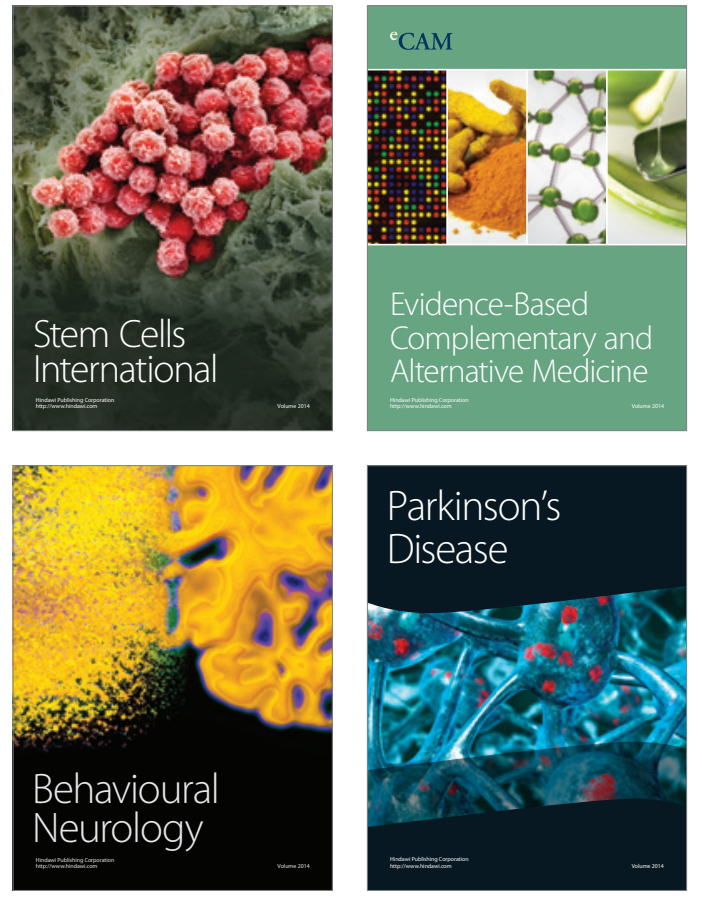
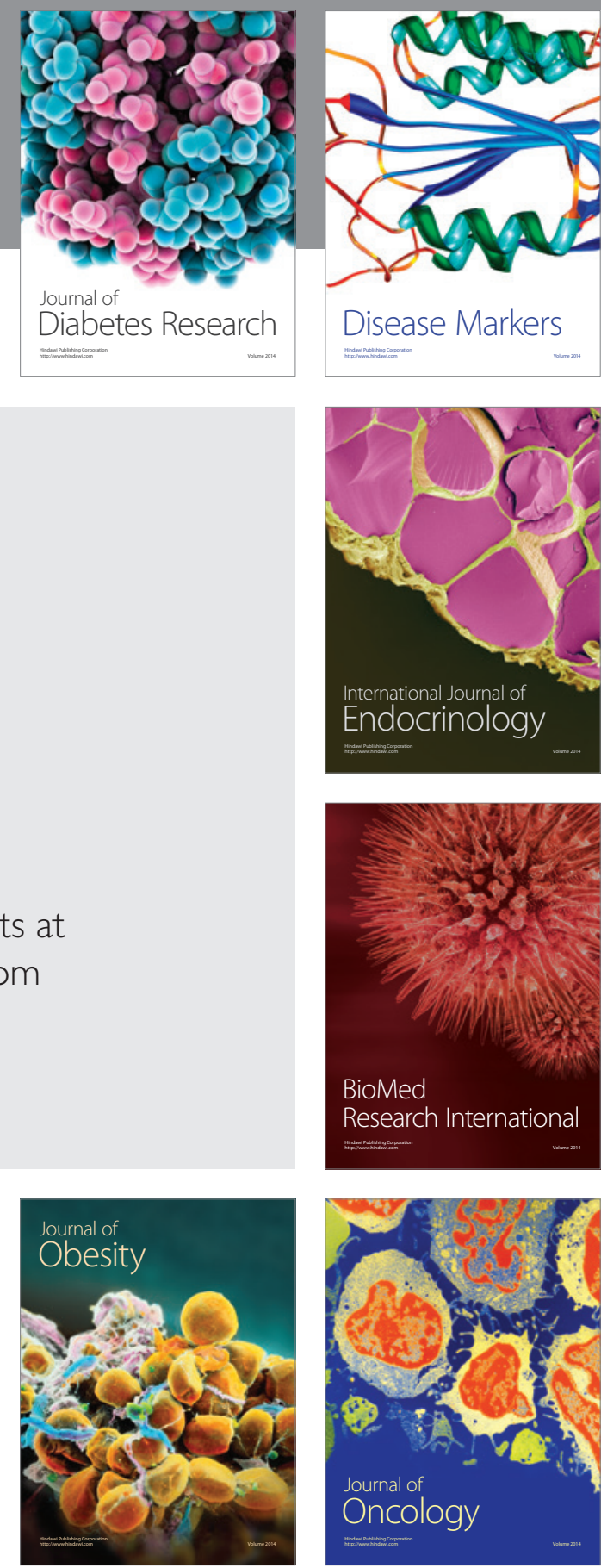

Disease Markers
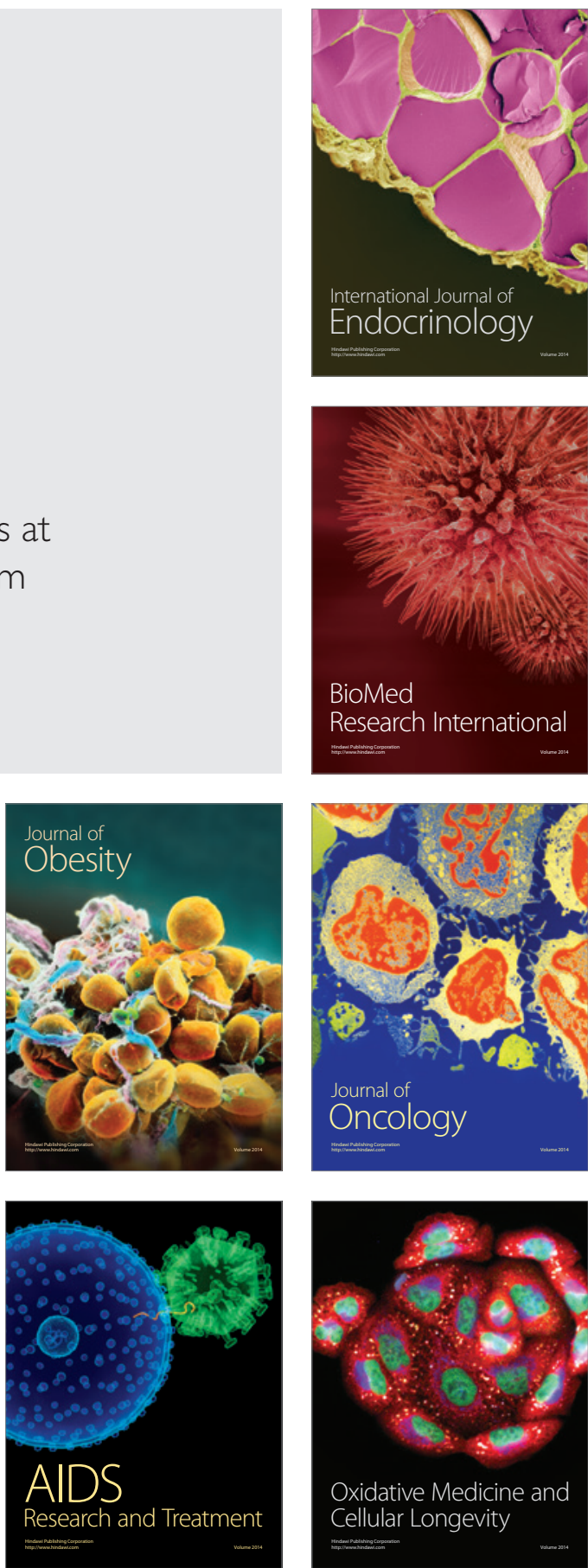